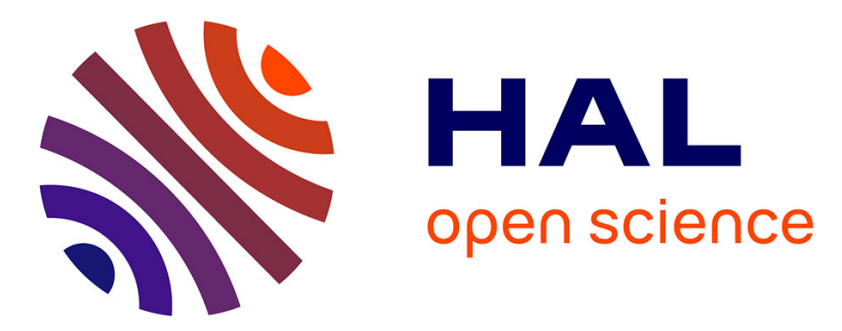

\title{
Gravity waves in Jupiter's equatorial clouds observed by the Galileo orbiter
}

\author{
J. Arregi, J.F. Rojas, R. Hueso, A. Sánchez-Lavega
}

\section{To cite this version:}

J. Arregi, J.F. Rojas, R. Hueso, A. Sánchez-Lavega. Gravity waves in Jupiter's equatorial clouds observed by the Galileo orbiter. Icarus, 2009, 202 (1), pp.358. 10.1016/j.icarus.2009.03.028 . hal00545292

\section{HAL Id: hal-00545292 \\ https://hal.science/hal-00545292}

Submitted on 10 Dec 2010

HAL is a multi-disciplinary open access archive for the deposit and dissemination of scientific research documents, whether they are published or not. The documents may come from teaching and research institutions in France or abroad, or from public or private research centers.
L'archive ouverte pluridisciplinaire HAL, est destinée au dépôt et à la diffusion de documents scientifiques de niveau recherche, publiés ou non, émanant des établissements d'enseignement et de recherche français ou étrangers, des laboratoires publics ou privés. 


\section{Accepted Manuscript}

Gravity waves in Jupiter's equatorial clouds observed by the Galileo orbiter

J. Arregi, J.F. Rojas, R. Hueso, A. Sánchez-Lavega

PII:

S0019-1035(09)00134-1

DOI: $\quad$ 10.1016/j.icarus.2009.03.028

Reference: $\quad$ YICAR 8978

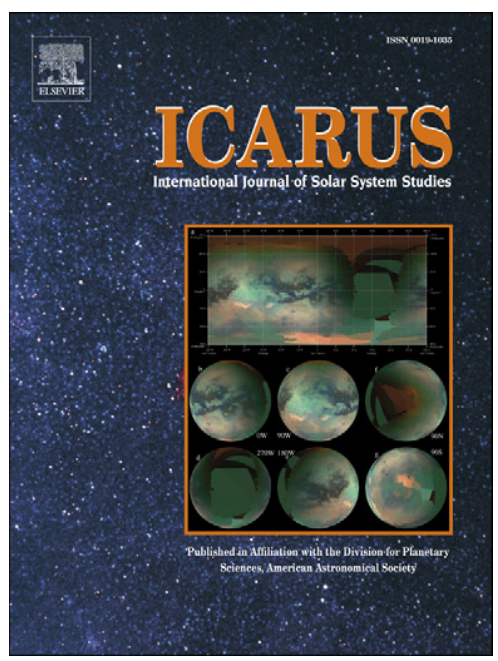

To appear in: Icarus

Received date: 18 November 2008

Revised date: 6 February 2009

Accepted date: 18 March 2009

Please cite this article as: J. Arregi, J.F. Rojas, R. Hueso, A. Sánchez-Lavega, Gravity waves in Jupiter's equatorial clouds observed by the Galileo orbiter, Icarus (2009), doi: 10.1016/j.icarus.2009.03.028

This is a PDF file of an unedited manuscript that has been accepted for publication. As a service to our customers we are providing this early version of the manuscript. The manuscript will undergo copyediting, typesetting, and review of the resulting proof before it is published in its final form. Please note that during the production process errors may be discovered which could affect the content, and all legal disclaimers that apply to the journal pertain. 


\section{GRAVITY WAVES IN JUPITER'S EQUATORIAL CLOUDS OBSERVED BY THE GALILEO ORBITER}

J. Arregi ${ }^{1}$, J. F. Rojas ${ }^{2}$, R. Hueso ${ }^{3}$ and A. Sánchez-Lavega ${ }^{3}$

${ }^{1}$ Departamento de Física Aplicada I. E.U.I.T.I. Vitoria, Universidad del País Vasco, Vitoria-Gazteiz (Spain)

${ }^{2}$ Departamento de Física Aplicada I. E.U.I.T.I. Bilbao, Universidad del País Vasco, Bilbao (Spain)

${ }^{3}$ Departamento de Física Aplicada I. E.T.S. Ingenieros, Universidad del País Vasco, Bilbao (Spain)

Manuscript pages $=12$

Figures $=2$

Tables $=1$ 


\begin{abstract}
A reanalysis of Galileo images of Jupiter's Equatorial Zone obtained in 1999 and 2001 reveals the presence of trains of cloud bands, probably generated by gravity waves, similar to those seen in Voyager 1 and 2 (1979) and New Horizons (2007) images. No such waves have been found in a similar analysis of the Cassini flyby (2000) dataset. We present the properties of these waves and discuss them in the context of previously proposed models and observations.
\end{abstract}

Key words: Jupiter, Atmosphere; Atmospheres, Dynamics 


\section{Introduction}

Gravity waves are a common feature on planetary atmospheres. One form they manifest is as trains of cloud bands when moisture favours condensation in lee waves forced by topography as observed on Earth and Mars (see e.g. Read and Lewis), on Venus clouds forced by deeper convection or wind shear instabilities (Peralta et al., 2008), and in Jupiter's upper cloud (Hunt and Muller, 1979; Flasar and Gierasch, 1986; Reuter et al., 2007). On cloud covered planets as Venus and Jupiter, gravity waves are an important tool to infer the properties of the buried atmosphere below clouds, as used for example in Jupiter by Flasar and Gierasch (1986).

In Jupiter, and chronologically, Voyagers' violet images at equatorial and near-equatorial latitudes showed alternating albedo bands spaced $300 \mathrm{~km}$ apart and arranged in wave-trains with the bands oriented along the North-South direction with about 15 front-lines (Hunt and Muller, 1979; Flasar and Gierasch, 1986). The short duration of the waves prevented the determination of their phase speeds. Next, the Galileo mission in 1996 showed similar wavetrains in violet images within the region of the Great Red Spot neighborhood (latitude $15^{\circ} \mathrm{S}$ ), but neither a characterization of these waves nor a systematic search for similar waves at the equator is present in the literature (Belton et al. 1996). On the other hand, no report of a search for gravity waves exists to our knowledge on Cassini images obtained during the Jupiter flyby in December 2000. During the New Horizons flyby in February 2007 a large number of wave-trains were detected by both the Long Range Reconnaissance Imager (LORRI) and Multispectral Visible Imaging Camera (MVIC) instruments fully covering the Jupiter equator (Reuter et al., 2007). Again the front lines had wavelengths close to $300 \mathrm{~km}$ and were N-S aligned (wave-trains running along parallel circles). Their measured phase 
speed with respect to the local zonal flow was $70 \mathrm{~m} / \mathrm{s}$ (Reuter et al, personal communication), which is an unexpectedly high value.

Due to these differences among the observed waves and their peculiarities, we have performed an in depth reanalysis of the Galileo and Cassini images to search in detail for the existence or lack of gravity waves in the equatorial region (similar to Voyager and New Horizons) and determine their characteristics for comparison with current models.

\section{Observations and measurements}

Our first aim was to know how the visibility of the waves (their contrast relative to surroundings) depends on the spatial resolution and observing wavelength (i.e. on the filters) used in the different missions. In Voyager images, gravity waves were detected in the violet filter $(416 \mathrm{~nm})$ with resolutions of $135 \mathrm{~km} /$ pixel (Hunt \& Muller, 1979; Flasar \& Gierasch, 1986). In images from New Horizons' MVIC instrument, waves were detected, and their phase speeds measured, in a narrow band methane filter $(860-910 \mathrm{~nm})$ at resolutions of 45 $\mathrm{km} /$ pixel. A visual inspection of MVIC images obtained in the wide band blue (400-550 nm), red (540-700 nm) and NIR (780-975) filters also shows the waves, althouth the images at these wavelengths do not cover the whole equatorial region. Also the LORRI-New Horizons panchromatic camera obtained images of these features with a spatial resolution of $11 \mathrm{~km} / \mathrm{pix}$ that were used to precisely determine their wavelength (Reuter et al., 2007).

We inspected Cassini images for the period of its closest approach to Jupiter from November 2000 to January 2001 and obtained with the filters UV3, BL1, CB2, CB3 and MT2 (filter details given in Porco et al., 2004). We were unable to find gravity waves near the Equator in any filter, in particular during the flyby's closest approach to Jupiter (30 December 2000) where the spatial resolution of the narrow angle camera images was $\sim 58 \mathrm{~km} / \mathrm{pix}$. The 
equatorial images with the highest resolution were cilyindrically projected at a spatial resolution of $0.05 \%$ pix in $\mathrm{MT} 2, \mathrm{CB} 3$ and BL1 filters and were carefully inspected, contrast stretched and processed with a variety of unsharp mask techniques and Butterworth digital filters searching unsuccessfully for possible waves. Also periodograms calculated from a cyclindrical map that covered the whole equatorial region and obtained from images acquired on 12 november 2000 at a spatial resolution of $110 \mathrm{~km}$ did not show any signature of shortscale waves. Although the Cassini image filters do not perfectly match those of Voyager and New Horizons, their wavelengths sense similar altitude levels corresponding to the tropospheric haze and the top of the ammonia cloud layer. The spatial resolution limit in Cassini images indicates that if the waves existed in the upper cloud with a contrast similar to the other spacecraft images, they should have been detected for wavelengths $>150 \mathrm{~km}$. The lack of detection is therefore not due to insufficient spatial or spectral resolution, neither due to an analysis of only small region of the Equatorial latitudes. When comparing the morphology of the Equatorial Zone in Cassini images with those of the other missions, it seems that it was more mottled and cloudier during the Cassini era, indicating perhaps more unstable conditions at the cloud level during that epoch that may have prevented wave formation.

However, our search for the waves in Galileo images of the equatorial region (where the resolutions were as fine as $\sim 22 \mathrm{~km} /$ pix in C20 images and $\sim 17 \mathrm{~km} /$ pix in I31 images) was successful. After careful image processing, we found wavy structures in two latitudes on 756 $\mathrm{nm}$ images corresponding to the C20 orbit (May 1999) and in another latitude on green images $(559 \mathrm{~nm})$ corresponding to the I31 orbit (August 2001) (see Figure 1). Overall, the spatial characteristics of these waves are similar to the ones previously reported in Voyager and New Horizons missions: near equatorial latitudes, near North - South orientation of the 
bands, variable packet length and width (below $1000 \mathrm{~km}$ ) and zonal wavelength between 100 and $200 \mathrm{~km}$ (see Table 1). To measure cloud motions, we reprocessed high resolution cylindrical projected images that were kindly provided by A. Vasavada, D. Banfield and M.T. Roman. The zonal phase speed velocity of the waves and the average zonal velocity of the flow were measured relative to System III in a series of images separated by $1.4-2 \mathrm{hr}$.

In figure 1 we also compare the phase velocity for the three wave packets with the mean zonal flow measured at these latitudes. The main uncertainty in the wavelength measurements comes from the fact that some wave bands are not straight and lack a uniform spacing between bands of the same wave-train. On the other hand, the uncertainty in the velocity arises from the limited spatial resolution of the images and from the reduced time span between them, although it was minimized by the availability of a series of images separated by 0.2 hours in the best cases. We performed a linear fit to the longitude-time plot for each crest (bright band) of the wave, obtaining from its slope the velocity. The error was estimated from the r.m.s of the speeds of the different wave crests and typical velocity uncertitudes are on the order of $8 \mathrm{~m} / \mathrm{s}$

These three wave packets showed motions similar to those previously found in Voyager images (Hunt \& Muller, 1979), but significantly different to those reported from New Horizons images (Reuter et al., 2007). For two wave packets, the phase velocities are within the calculated error bars of the mean zonal flow (they are therefore quasi-stationary), whereas the phase speed of the third wave packet is clearly higher than the mean zonal flow, although still much lower than that reported from New Horizons. Also New Horizons images showed that wave activity extended over a large area of the equatorial region when compared with Voyager and Galileo data sets. 


\section{Interpretation}

As done in previous studies of the Voyager and New Horizons waves, we interpret the Galileo waves as gravity waves due to their properties (Table 1) and their visual similarity to classical lee waves forming by topography on Earth and Mars. According to a simple linear model that assumes no vertical wind shear and constant Brunt-Väisälä frequency (Holton, 2004), the dispersion relationship for gravity waves is

$$
c_{x}-\left\langle u>= \pm \frac{N}{\sqrt{k^{2}+m^{2}}}\right.
$$

where $c_{x}-\langle u\rangle$ is the phase speed of the gravity wave relative to the mean zonal flow velocity, $N$ is the Brunt-Väisälä frequency, $k=2 \pi / L_{x}$ and $m=2 \pi / L_{z}$ are the horizontal and vertical wavenumbers ( $L_{x}$ and $L_{z}$ the respective wavelengths). The Doppler shifted frequency of the waves $v=\omega / 2 \pi=c_{x}-<u>/ L_{x}$ must be $<N$ for vertical wave propagation $\left(m^{2}>0\right)$. To calculate the phase speed we measured in the same images the local zonal wind profile in the $0-4^{\circ} \mathrm{N}$ latitude range (see Figure 1 ). Our measurements compare well with values obtained for a few degrees South $\left(0-4^{\circ} \mathrm{S}\right)$ during the Galileo mission (Vasavada et al. 1998) and is in accordance within the error bars with winds derived during the Cassini flyby. The worst case for non-vertical propagation corresponds to the wave packet at $2.8^{\circ}$ latitude, which has the large phase speed. For this packet we have $v=1.9 \times 10^{-4} \mathrm{~s}^{-1}$ and this value is taken as a lower limit for the buoyancy frequency in equation (1). Note that at the low latitude of these waves the Coriolis frequency $f=2 \Omega \sin \varphi \sim 10^{-5} \mathrm{~s}^{-1}<v$ (here $\Omega$ is Jupiter's rotational frequency and $\varphi$ is the latitude), indicating that they can be treated as pure gravity waves (not as inertiogravity waves, Holton, 2004). 
Figure 2 presents a plot of the dispersion relationship calculated for a typical tropospheric value of $N=5 \cdot 10^{-3} \mathrm{~s}^{-1}$ as measured at 0.5 bar in a nearby region (latitude $7^{\circ} \mathrm{N}$ ) during the Galileo probe descent (Magalhaes et al., 2002). The vertical wavelength is left as a free parameter to fit to the observations. Accordingly, the two waves close to the Equator should have vertical wavelengths of roughly $10 \mathrm{~km}$ or half the atmospheric scale height. The waves at latitude $2.8^{\circ} \mathrm{N}$ fit for a vertical wavelength of $45 \mathrm{~km}$ or about 2 scale heights.

Detailed measurements of the temperature field during the descent of the Galileo probe in 1995 at $7^{\circ} \mathrm{N}$ (starting at the pressure level 0.47 bar) detected a region of high static stability between pressure levels of 0.5 and 1.7 bar, and oscillatory structures in the temperature profile with amplitudes $\sim 0.1-0.2 \mathrm{~K}$ that showed vertical wavelengths of $\sim 15-30 \mathrm{~km}$ (Magalhães et al., 2002). These authors interpreted the oscillations in a modified linear model as gravity waves with horizontal phase speed $c_{x}=160 \mathrm{~ms}^{-1}$. At $7^{\circ} \mathrm{N}$ the mean zonal wind is $<u>=110 \mathrm{~ms}^{-1}$ giving $c_{x}-<u>=50 \mathrm{~ms}^{-1}$. These numbers (vertical wavelength and Doppler shifted phase speed) fit reasonably well our data for the $2.8^{\circ} \mathrm{N}$ latitude wave packet. Young et al. (2005) also assigned the Galileo probe temperature oscillations above cloud level (amplitudes $\sim 3-6 \mathrm{~K}$ and vertical wavelengths of $\sim 10-24 \mathrm{~km}$ ) as damped and undamped gravity waves.

Flasar and Gierasch (1986) interpreted the Voyager mesoscale wave packets as ducted gravity waves trapped below 0.7 bar by an uppermost low static stability layer between 0.5 and 0.7 bar. No phase speed was used to constraint the altitudes, but their model predictions are qualitatively consistent with our observations. Bosak and Ingersoll (2002) proposed an alternative interpretation of the Voyager waves based on a vertical shear instability model of the zonal wind. They used the measurements by the Galileo probe at $7^{\circ} \mathrm{N}$ of the vertical shear of the zonal wind (it must be remembered that the probe entered a peculiar region of Jupiter 
known as a "Hot Spot" so conditions there may not be representative of nearby latitudes in Jupiter). The model predicts a dispersion relationship for the waves with phase speeds increasing with decreasing wavelengths (see their figures 6-8). Accordingly, for a sheared unstable layer $46 \mathrm{~km}$ thick, waves with a wavelength of a zonal $100-200 \mathrm{~km}$ should have Doppler shifted phase speeds of $\sim 32 \mathrm{~ms}^{-1}$, also in reasonable agreement with our data for the $2.8^{\circ}$ wave packet. However, for this to occur the buoyancy frequencies must be an order of magnitude lower $\left(N \sim 5 \times 10^{-4} \mathrm{~s}^{-1}\right)$ than those we retrieve. With such values the linear gravity wave dispersion relationship (1) predicts vertical wavelengths $\sim 1000 \mathrm{~km}$, that are unrealistic. Accordingly we favour the Flasar and Gierasch interpretation but it must be mentioned that our interpretation in terms of a linear model could also be very simplistic so Bosak and Ingersoll (2002) interpretation cannot be disregarded from this study. .

The variability detected in mesoscale gravity wave activity in the equatorial region of Jupiter over this large period, i. e. high in New Horizons, scarce in Voyager and Galileo, and non existent in Cassini, probably reflects the changing conditions in the unstable layer where they are forced or in the stable layer where they propagate. The mottled and cloudy aspect of the Equatorial Zone during the Cassini period points toward a particularly unstable situation of the layer where the waves form (extending vertically between 0.1 and 0.5 bar). A detailed model of gravity waves in Jupiter's equatorial region that assimilates all the data gathered so far could help to understand the structure of the upper equatorial troposphere and its spatial and temporal variability.

\section{Acknowledgements}

We acknowledge M. Roman, D. Bandfield and A. Vasavada for providing us with cylindrically projected images from the Galileo spacecraft. This work has been funded by 
Spanish MEC AYA2006-07735 with FEDER support and Grupos Gobierno Vasco IT-464-

07. RH acknowledges a "Ramón y Cajal” contract from MEC. 


\section{REFERENCES}

Belton, M.J.S. and 33 colleagues, 1996. Galileo's first images of Jupiter and the Galilean satellites. Science, 274, 377-379.

Bosak, T. and Ingersoll, A.P., 2002. Shear instabilities as a probe of Jupiter's atmosphere. Icarus, 158, 401-409

Flasar, F.M. and Gierasch P.J., 1986. Mesoscale waves as a probe of Jupiter's deep atmosphere. J. Atmos. Sci, 43, 2683-2707.

Holton, J.R., 2004. An introduction to dynamic meteorology. $4^{\text {th }}$ edition. Academic Press Inc.

Hunt, G.E., and Muller, J. P., 1979. Voyager observations of small-scale waves in the equatorial region of the jovian atmosphere, Nature, 280, 778-780.

Lindal, G.F. et al., 1981. The atmosphere of Jupiter: An analysis of the Voyager radio occultation measurements, J. Geophys. Res., 86(A10), 8721-8727.

Magalhães, J.A. et al., 2002. The stratification of Jupiter's troposphere at the Galileo probe entry site. Icarus $\mathbf{1 5 8}, 410-433$.

Peralta, J., Hueso R., Sánchez-Lavega A., Piccioni G., Lanciano O., and Drossart, P., 2008. Characterization of mesoscale gravity waves in the upper and lower clouds of Venus from VEX-VIRTIS images. Journal Geophysical Research-Planets, 113, E00B18 doi:10.1029/2008JE003185.

Porco, C.C. and 20 colleagues, 2004. Cassini imaging science: instrument characteristics and anticipated scientific investigations at Saturn. Space Science Reviews 115, 363 - 497.

Read, P.L. and Lewis, S.R. 2004. The Martian Climate Revisited. Springer-Praxis, Chichester (UK). 
Reuter, D.C. et al., 2007. Jupiter cloud composition, stratification, convection and wave motion: A view from New Horizons. Science, 318, 223-225.

Salby, M.L., 1996. Fundamentals of Atmospheric Physics. Academic Press Inc.

Vasavada, A. R., 1998. Galileo Imaging of Jupiter's Atmosphere: The Great Red Spot, Equatorial Region, and White Ovals. Icarus, 135, 265-275.

Young, L.A. et al., 1997.Gravity waves in Jupiter's thermosphere. Science, 276, 108-110.

Young, L.A. et al. 2005. Gravity waves in Jupiter's stratosphere as measured by the Galileo ASI experiment. Icarus, 173, 185-199. 


\section{Figure Captions}

\section{Figure 1:}

Upper image: Waves in orbit C20 (May 1999) from Galileo $756 \mathrm{~nm}$ images Middle image: Wave in orbit I31 (August 2001) from green (559 nm) Galileo images. The area of the waves has been contrast enhanced and is enclosed by rectangles. Lower graph: mean zonal wind velocity from a fit to individual single point measurements, and phase speed of the three waves relative to System III. The zonal wind velocity profile has been obtained from datapoints binned each $0.2^{\circ}$ and then fitted with a second degree polinomial.

\section{Figure 2:}

Gravity wave dispersion relationship from a linear model in the shortwave limit (Holton, 2004; Salby, 1996). The continuous curves have been calculated for a value of the BruntVäisääla frequency $N=5 \cdot 10^{-3} \mathrm{~s}^{-1}$ and for five values of the vertical wavelength $\left(L_{z}\right)$. The values for the three waves are also plotted as single points with measured error bars. Errors in $\mathrm{k}$ have been evaluated from errors in measuring zonal wavelength. 
TABLE 1

Summary of wave packets properties observed.

\begin{tabular}{|c|c|c|c|}
\hline Parameter & WAVE 1 & WAVE 2 & WAVE 3 \\
\hline North planetocentric latitude $\left(^{\circ}\right)$ & $2.8 \pm 0.1$ & $0.18 \pm 0.02$ & $1.82 \pm 0.03$ \\
\hline Packet length $(\mathrm{km}) \mathrm{E}-\mathrm{W}$ & $760 \pm 25$ & $210 \pm 25$ & $335 \pm 25$ \\
\hline Packet width $(\mathrm{km}) \mathrm{N}-\mathrm{S}$ & $435 \pm 25$ & $995 \pm 25$ & $525 \pm 25$ \\
\hline Wavelength $(\mathrm{km})$ & $180 \pm 25$ & $205 \pm 10$ & $110 \pm 20$ \\
\hline Orientation angle $\left(^{\circ}\right)$ & $-20 \pm 7$ & $2 \pm 3$ & $-21 \pm 5$ \\
\hline Number of wave bands & 5 & 6 & 4 \\
\hline$c-<u>(\mathrm{m} / \mathrm{s})$ & $35 \pm 8$ & $8 \pm 8$ & $0 \pm 8$ \\
\hline
\end{tabular}


FIGURE 1
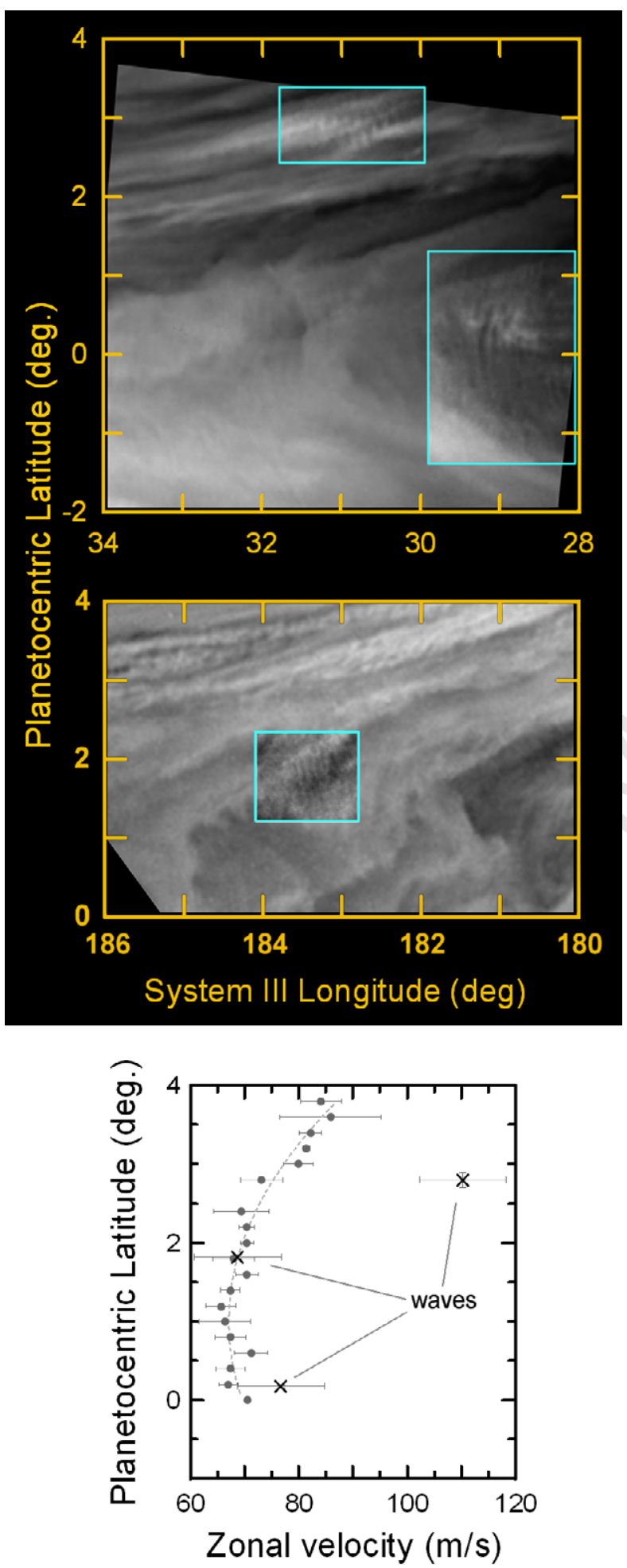
FIGURE 2

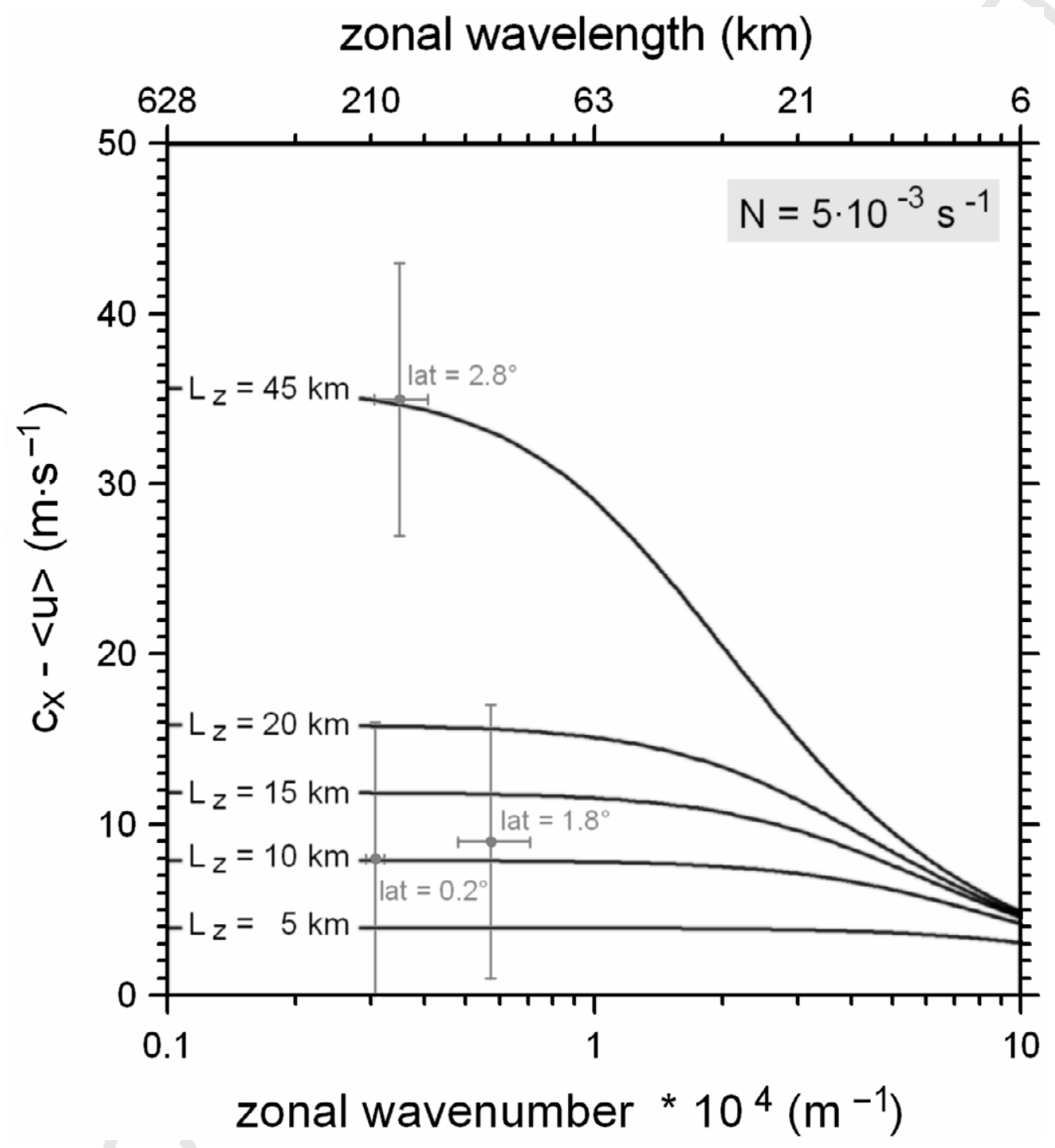

